\title{
Alternative sputum collection methods for diagnosis of childhood intrathoracic tuberculosis: a systematic literature review
}

\author{
Vincent loos, ${ }^{1}$ Hugues Cordel, ${ }^{2}$ Maryline Bonnet ${ }^{3}$
}

\begin{abstract}
- Additional material is published online only. To view please visit the journal online (http://dx.doi.org/10.1136/ archdischild-2018-315453).
\end{abstract}

${ }^{1}$ Département Médical, Médecins Sans Frontières, Paris, Paris, France

${ }^{2}$ Infectious disease department, Avicenne Hospital, Bobigny, Seine-Saint-Denis, France ${ }^{3}$ IRD UMI233 TransVIHMI-UMINSERM U1175, Montpellier, France

\section{Correspondence to} Dr Maryline Bonnet, Institut de recherche pour le developpement France-Sud, UMI 233 IRD - U1175 INSERM - Université de Montpellier, Montpellier 34394, France maryline.bonnet@epicentre. msf.org

Received 2 May 2018 Revised 3 July 2018 Accepted 8 July 2018 Published Online First 20 August 2018

\section{Linked}

- http://dx.doi.org/10.1136/ archdischild-2018-315411

Check for updates

(C) Author(s) (or their employer(s)) 2019. No commercial re-use. See rights and permissions. Published by BMJ.

To cite: loos V, Cordel $\mathrm{H}$ Bonnet M. Arch Dis Child 2019;104:629-635.

\begin{abstract}
Background Diagnosis of intrathoracic tuberculosis (ITB) is limited in children partly by their difficulty to produce sputum specimen.
\end{abstract}

Objective To systematically review the detection yields of mycobacterial culture and Xpert MTB/RIF from induced sputum (IS), nasopharyngeal aspirate (NPA) and gastric aspirate (GA) in children with presumptive ITB.

Design Pubmed, Embase and Biosis databases and grey literature were searched. Randomised controlled trials, cohort, cross-sectional or case control studies using IS, GA and NPA for diagnosis of ITB published between January 1990 and January 2018 were included. Data were extracted on study design, case definition of presumptive ITB, sample collection methods, outcome measures and results.

Results 30 studies were selected, including 11554 children. Detection yields for culture ranged between $1 \%$ and $30 \%$ for IS, $1 \%$ and $45 \%$ for GA and $4 \%$ and $24 \%$ for NPA. For Xpert MTB/RIF, it was between $2 \%$ and $17 \%$ for $\mathrm{IS}, 5 \%$ and $51 \%$ for GA and $3 \%$ and $8 \%$ for NPA. There was a tendency of better yields with IS when the pretest probability of ITB was low to moderate and with GA when it was high. Sampling a second specimen contributed for $6 \%-33 \%$ of the cumulative yield and combination of different methods significantly increase the detection yields.

Conclusions Despite the important study heterogeneity, any of the specimen collection methods offers good potential to confirm childhood ITB. However, their operational challenges were poorly evaluated. In the absence of a sensitive non-sputum based test, only a minority of children with ITB can be confirmed.

\section{INTRODUCTION}

Childhood intrathoracic tuberculosis (ITB) is challenging to diagnose, because the disease is often paucibacillary and respiratory secretions are difficult to collect. ${ }^{1}$ There have been a lot of efforts to improve detection of Mycobacterium tuberculosis in biological samples. In children, the Xpert MTB/RIF assay (Xpert; Cepheid, Sunnyvale, USA) allows rapid diagnostic of two-thirds of children with culture positive samples. ${ }^{2}$ Unfortunately, due to the absence of diagnostic reference standard of childhood ITB, majority of patients are treated empirically on the basis of clinical or radiological suspicion. ${ }^{3}$ Consequently, many diagnosis accuracy studies of childhood ITB only look at the small part of microbiologically confirmed cases.

\section{What is already known on this topic?}

Diagnosis of intrathoracic tuberculosis uses sputum specimens that are difficult to collect in children, who are also more likely to present paucibacillary disease.

- Only a small proportion of childhood intrathoracic tuberculosis cases are microbiologically confirmed.

- Xpert can detect two-thirds of children with culture positive samples but is limited by the difficulty to collect specimen, mainly in lowincome and middle-income countries.

\section{What this study adds?}

- There is high variability of tuberculosis detection yields from alternative sputum specimen collection methods mainly because studies use different definition of presumptive intrathoracic tuberculosis.

- Combination of different specimen collection methods on the same day is probably as effective as repeated sampling on consecutive days with the same method.

- Operational considerations and training strategy are essential in choosing the appropriate collection method for implementation at low health facility level.

In high burden low-income and middle-income countries (LMIC), TB in children remains underdiagnosed with only $45 \%$ of the estimated number of cases notified to the WHO in $2016^{4}$. In many of these countries, rising levels of drug resistance makes it essential to achieve the highest possible rates of microbiological confirmation in order to tailor the treatment.

To optimise the performance of microbiological tests on respiratory samples, it is critical to select the best specimen collection method, especially in children who have difficulties in producing sputum spontaneously. ${ }^{1}$ The most advanced method, the bronchoalveolar lavage, is not available in many LMIC. Other methods, such as induced sputum (IS), gastric aspirate (GA) or nasopharyngeal aspirate (NPA) are potential good alternatives.

In order to support clinicians and TB programmes in choosing the best method, our study systematically reviewed the value of alternative sputum 
collection methods to achieve microbioogical confirmation of ITB in children from LMIC. Our main objective was to determine and compare the diagnostic yield of IS, NPA and GA using culture and Xpert in children with presumptive ITB. Our secondary objective was to determine the proportion of additional cases detected when collecting several samples and the value of combining different methods.

\section{METHODS}

\section{Search strategy and selection criteria}

Randomised controlled trials, cohort, cross-sectional or casecontrol studies published between January 1990 and f 2018 were included. Reviews, opinion papers and case reports were excluded. Studies from LMIC (World Bank definition) investigating the yield of GA, NPA or IS sampled for culture or Xpert were considered for inclusion. Expectorated samples (ES) were excluded from this review but studies investigating ES could be included if GA, NPA or IS were simultaneously evaluated and separate results were presented. Study participants aged less than 15 years tested for presumptive ITB were included. Presumptive ITB was defined by the presence of at least one symptom suggestive of tuberculosis. Studies that included asymptomatic patients (child contacts or children participating in a vaccination trial or prevalence survey), children investigated for acute pneumonia and children on antituberculosis treatment were excluded.

We conducted systematic search of scientific publications and grey literature in the scientific databases Embase, Medline/ Pubmed, BIOSIS and New York Academy of Medicine Grey Literature Report. The key words used were gastric aspirate, induced sputum, nasopharyngeal aspirate, tuberculosis, child and their synonyms. Identification and screening of the records on the basis of their title/abstract was done by a single author (VI). In case no abstract was available, the study was considered as potentially eligible based on the title only. Full-text articles were reviewed independently by two authors (HC and VI) for study eligibility and by a third reviewer (MB) in case of discordance. Reasons for excluding studies were documented.

\section{Data extraction and analysis}

Data on main/secondary outcomes, study, patients and index tests characteristics (age, HIV status, inpatients/outpatients, chest X-ray (CXR) findings) were collected through a structured data collection form separately by HC and VI and discrepancies were discussed with MB. Methodological quality of eligible studies was assessed with the Quadas-2 assessment tool that includes four domains: patient selection, index test, reference standard, flow and timing. Because of the absence of a reference standard of childhood ITB, the items related to reference standard and $2 \times 2$ tables were omitted. Signalling questions were added to address the methodological issues of studies comparing index tests between them. It resulted in an 'adapted Quadas-2' (online Supplementary table 1).

After a preliminary review of included studies and before systematic data extraction, we identified nine criteria commonly used to define 'presumptive ITB': symptom complex suggestive of ITB (>1 symptom without specification), cough more than 2 weeks, weight loss or failure to gain weight, fever more than 1 or 2 weeks, painless swelling of superficial nodes, TB contact history, CXR suggestive of ITB or abnormal CXR, positive tuberculin skin test, persistence of symptoms or CXR abnormalities after empirical antibiotic treatment. We defined low pretest probability of ITB by the presence of at least one clinical sign or symptom; moderate probability by the presence of at least two criteria (including at least one clinical sign or symptom OR presence of the criteria 'symptom complex suggestive of tuberculosis') and high probability by the presence of at least three criteria including at least one clinical sign or symptom OR two criteria including one clinical sign or symptom and persistence of symptoms or CXR abnormalities after empirical antibiotic treatment. If there was no information on the definition of 'presumptive ITB' or if the definition included less than three criteria out of eight criteria (excluding 'symptom complex suggestive of tuberculosis') preventing the classification of high probability, the pretest probability was qualified as 'indeterminate'. We also collected, whenever reported, the number of adverse events per procedure and per patient.

Quantitative data and quality assessments ('adapted Quadas2') were entered in RevMan V.5.3 to generate descriptive statistics, figures and tables. The yield of a sample collection method for a diagnostic test (Xpert and culture) was defined as the number of patients with a positive test out of the total number of patients tested. Because of the absence of reference standard, sensitivity and specificity could not be calculated. Detection yields with exact binomial 95\% CI are presented according to the pretest probability of ITB. Additional yields of second specimen (ie, number of positive patients in the second specimen while being negative on the first) are presented in absolute percentage of study participants.

The study protocol was registered in PROSPERO (International Prospective Register of Systematic Reviews) under the number CRD42014012924.

\section{RESULTS}

\section{Studies characteristics}

Out of 440 records screened, 30 studies (31 articles) ${ }^{5-35}$ were included in the review (online Supplementary figure 1).

The total number of study participants was 11554 . The greater number of patients were from India $(\mathrm{n}=3098,27 \%)^{11} 1214-1633$ and South Africa $(n=3312,29 \%) .{ }^{10} 18-202227293235$ There was great heterogeneity of definitions of presumptive ITB and patient characteristics across studies (table 1). Most studies were done in tertiary care centres and only two ${ }^{20} 32$ in primary care centres. Half of the studies included inpatients and outpatients. Pretest probability of ITB was estimated as 'moderate' in half of the studies and could not be assessed in four studies ${ }^{15} 232528$ (online Supplementary table 2). In 23 (77\%) studies, majority of children were aged $<5$ years. When reported, proportion of HIV patients across studies varied from $8 \%{ }^{20}$ to $70 \%{ }^{8}$ Culture, Xpert and both were used in 29, 13 and 12 studies, respectively. Solid culture media was used in nine studies, liquid media in 11 and both media in seven studies.

\section{Specimen collection procedures}

GA was performed early morning, after an overnight or 4-8 hours fast. Only two studies ${ }^{29} 30$ reported the minimum volume of gastric liquid aspirated: $10 \mathrm{~mL}$ for the first and $20 \mathrm{~mL}$ for the second. Four studies ${ }^{16252933}$ reported the use of a normal saline wash to collect gastric liquid and in two studies, the use of sterile water in case of aspiration of less than $3 \mathrm{~mL}$ of gastric fluid. ${ }^{727}$ NPA was preceded by instillation of normal saline in three studies ${ }^{71920}$ but not in two studies. ${ }^{5}$ IS procedure was well described and standardised in 19/20 studies (online Supplementary table 3). Main differences were the type of nebulizer used (jet vs ultrasonic), the concentration of the hypertonic saline (3 vs 5\%) and use of physiotherapy to help induction. Physiotherapy was performed in nine studies. In 16 studies, 


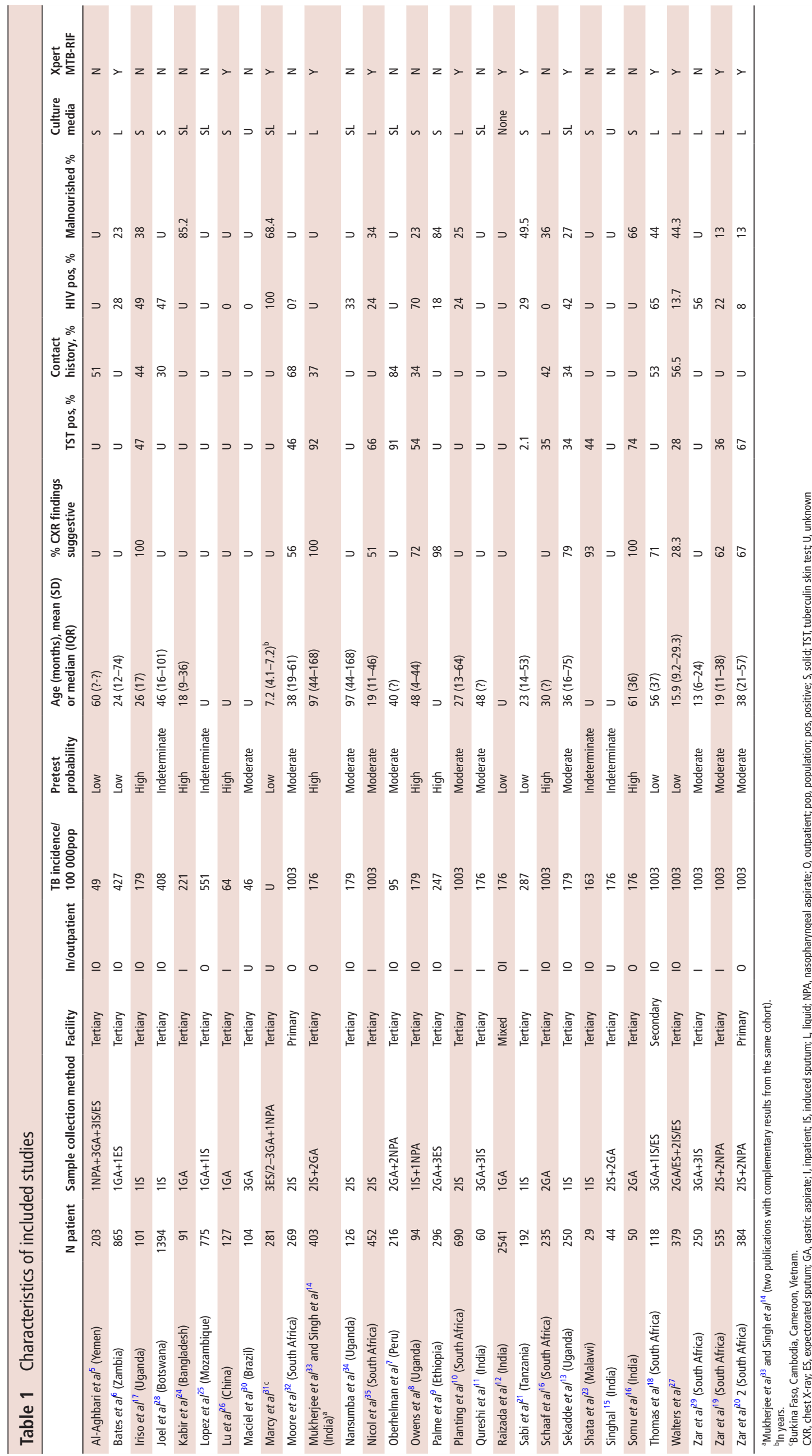


Table 2 Comparison of culture or Xpert MTB/RIF detection yield or sensitivity between different sputum collection methods in same patients

\begin{tabular}{|c|c|c|c|c|c|c|c|}
\hline Studies & $\mathbf{N}$ & Pretest probability & Methods & Test & Difference in yields $(\%)(95 \% \mathrm{Cl})$ & Difference in sensitivity $(\%)(95 \% \mathrm{Cl})$ & $P$ values \\
\hline Zar et al ${ }^{29 *}$ & 250 & Moderate & $2 \mathrm{IS}$ vs $2 \mathrm{GA}$ & Culture & $\begin{array}{l}+5.3 \%, 15 \% \\
(11 \text { to } 20) \text { vs } 8 \% \text { ( } 5 \text { to } 12 \text { ) }\end{array}$ & & $0.018 \dagger$ \\
\hline Qureshi et al ${ }^{11}$ & 60 & Moderate & $3 \mathrm{IS}$ vs $3 \mathrm{GA}$ & Culture & $\begin{array}{l}+5 \%, 13 \% \\
\text { ( } 3 \text { to } 18) \text { vs } 8 \% \text { ( } 6 \text { to } 25 \text { ) }\end{array}$ & & \\
\hline Mukherjee et $a^{33 *}$ & 403 & High & $2 \mathrm{IS}$ vs $2 \mathrm{GA}$ & Culture & $\begin{array}{l}-15 \%, 18 \% \\
\text { (14 to } 22) \text { vs } 33 \% \text { (28 to } 37 \text { ) }\end{array}$ & & $<0.001 \dagger$ \\
\hline Singh et al ${ }^{14 *}$ & 130 & High & $2 \mathrm{IS}$ vs $2 \mathrm{GA}$ & Xpert & $\begin{array}{l}-10 \%, 22 \% \\
\text { (15to } 30) \text { vs } 32 \% \text { ( } 24 \text { to } 40)\end{array}$ & $\begin{array}{l}+2.5 \%, 70 \% \\
\text { (56.2 to } 80.9 \text { ) vs } 67.5 \% \text { ( } 56.5 \text { to } 76.9 \text { ) }\end{array}$ & 0.770 \\
\hline Owens $^{8 *}$ & 94 & High & $1 \mathrm{IS}$ vs $1 \mathrm{NPA}$ & Culture & $\begin{array}{l}-2 \%, 22 \% \\
\text { (14 to } 32 \text { ) vs } 24 \% \text { (15 to } 34 \text { ) }\end{array}$ & & $>0.5 \dagger$ \\
\hline Zar et l $^{19 *}$ & 396 & Moderate & $2 \mathrm{IS}$ vs $2 \mathrm{NPA}$ & Culture & $\begin{array}{l}+3 \%, 15 \% \\
(12 \text { to } 19) \text { vs } 12 \% \text { ( } 9 \text { to } 16)\end{array}$ & & $<0.01$ \\
\hline Zar et $a l^{*}$ & 396 & Moderate & 215 vs $2 N P A$ & Xpert & $\begin{array}{l}+1 \%, 9 \% \\
\text { (6 to } 12) \text { vs } 8 \%(5 \text { to } 12)\end{array}$ & $\begin{array}{l}+6.3 \%, 71.4 \% \\
\text { (60.0 to } 82.9) \text { vs } 65.1 \%(53.0 \text { to } 77.2)\end{array}$ & 0.440 \\
\hline Zar et $a^{20 *}$ & 389 & Moderate & $2 \mathrm{IS}$ vs $2 \mathrm{NPA}$ & Xpert & $\begin{array}{l}+1 \%, 4 \% \\
\text { (2 to } 7) \text { vs } 3 \%(1 \text { to } 5)\end{array}$ & $\begin{array}{l}+18 \%, 57 \% \\
\text { (39 to } 75) \text { vs } 39 \% \text { ( } 24 \text { to } 58)\end{array}$ & 0.180 \\
\hline Oberhelman et $a l^{7 *}$ & 216 & Moderate & $2 \mathrm{GA}$ vs $2 \mathrm{NPA}$ & Culture & $\begin{array}{l}+5 \%, 10 \% \\
\text { (6 to } 15) \text { vs } 6 \% \text { (3 to } 10)\end{array}$ & & $<0.05 t$ \\
\hline Al-Aghbari ${ }^{5}$ & 203 & Low & $1 \mathrm{GA}$ vs $1 \mathrm{NPA}$ & Culture & $\begin{array}{l}+2 \%, 9 \% \\
\text { (5 to } 14 \text { ) vs } 7 \% \text { (4 to } 11)\end{array}$ & & \\
\hline
\end{tabular}

*Paired samples.

†Exact McNemar's test.

GA, gastric aspirate; IS, induced sputum; NPA, nasopharyngeal aspirate.

bronchodilators were administered before induction and in eight studies samples were collected after nasopharyngeal suction. In Moore et al, ${ }^{32}$ Planting et $a l^{10}$ and Sabi et al, ${ }^{21} 60 \%, 21 \%$ and $1.6 \%$ of samples, respectively, were expectorated spontaneously after IS.

\section{Detection yields}

Yield of Xpert and culture according to specimen collection method and pretest probability of ITB are reported in online Supplementary figures 1 and 2 and online Supplementary tables 4 and 5 . The detection yield (first sample) for culture ranged between $1 \%$ and $30 \%$ for IS, $1 \%$ and $45 \%$ for GA and $4 \%$ and $24 \%$ for NPA across studies. For Xpert, it was between $2 \%$ and $17 \%$ for IS, $5 \%$ and $51 \%$ for GA and $3 \%$ and $8 \%$ for NPA. In Lu et al, ${ }^{26}$ Xpert detected much more cases than culture (51.2 vs $19.7 \%$ ) from the same GA sample.

Using 10\% threshold of detection yield in patients with low to moderate pretest probability, the proportion of studies reporting yield of culture and Xpert $>10 \%$ tended to be higher for IS $(7 / 12,58.3 \%$ and $2 / 8,25.0 \%)$ than for GA $(1 / 7,14.3 \%$ and $0 / 3)$ or NPA $(1 / 4,25.0 \%$ and $0 / 3)$. When the pretest probability was high, using a threshold of detection yield of $20 \%$, the proportion of studies with culture detection yield $>20 \%$ was similar betwenn GA and IS $(4 / 6,66.7 \%$ vs $2 / 3,66.7 \%)$ and tended to be higher for GA with Xpert (2/2 vs 0/1). Yield of culture performed on NPA was available from one study ${ }^{8}$ with high probability of ITB $(24 \%)$. In primary care setting, IS had a lower detection yield than in tertiary setting. ${ }^{20} 32$

Nine studies 578111419202933 presented the detection yields of different specimen collection methods in same patients (table 2). Four studies ${ }^{11} 142933$ compared IS and GA, three using culture $^{112933}$ and one using Xpert. ${ }^{14}$ Detection yield was higher for GA in two studies with high pretest probability and for IS in two studies with moderate pretest probability of ITB. Of three studies comparing detections yields between IS and NPA, two favoured IS with a very low difference in yields ranging between $1 \%$ and $3 \%$, all in patients with moderate probability of ITB.
However, the difference was significant in the Zar et al's ${ }^{19}$ study with 396 patients. In the study ${ }^{8}$ among children with high pretest probability, NPA had a slight better culture (+2\%) detection yield but the difference was not significant. Only two studies ${ }^{57}$ compared GA with NPA, with a trend for better culture detection yield for GA.

Sampling a second specimen contributed for $6 \%-10 \%$ of the cumulative yield in children with low pretest probability and for $6 \%-33 \%$ in children with moderate or high probability of ITB, regardless of the specimen collection method and microbiological test used (figures 1 and 2).

Seven studies assessed the detection yield on combining different sample collection methods (table 3). The contribution of one method alone ranged between $66 \%$ and $100 \%$ for GA, $49 \%$ and $95 \%$ for IS and 55\% and $87.5 \%$ for NPA. In three studies, combining 2-3 methods on the first day detected between $74 \%$ and $100 \%$ of cases detected over $2-3$ consecutive days.

\section{Tolerability}

Only seven studies ${ }^{10} 182128293234$ reported tolerability data and only for the IS. The proportion of patients with adverse events ranged between $0.4 \%$ and $27.9 \%$ for nose bleeding, $0 \%-1.1 \%$ for wheezing, $0 \%-1.6 \%$ for transient hypoxia and $0.5 \%-4.0 \%$ for vomiting. In one study, ${ }^{10} 14$ (2\%) children required bronchodilator after the procedure due to wheezing that was reversible in all children after bronchodilator. In this study, median oxygen saturation was 98\% (IQR 97-98) before IS (online Supplementary table 6)

\section{DISCUSSION}

Our systematic review confirms that culture and Xpert yields for detection of ITB are low in children with presumptive ITB and shows conflicting data on comparison of detection yields between GA, IS and NPA. As expected, the detection yields are correlated to the pretest probability of ITB that is related to the 


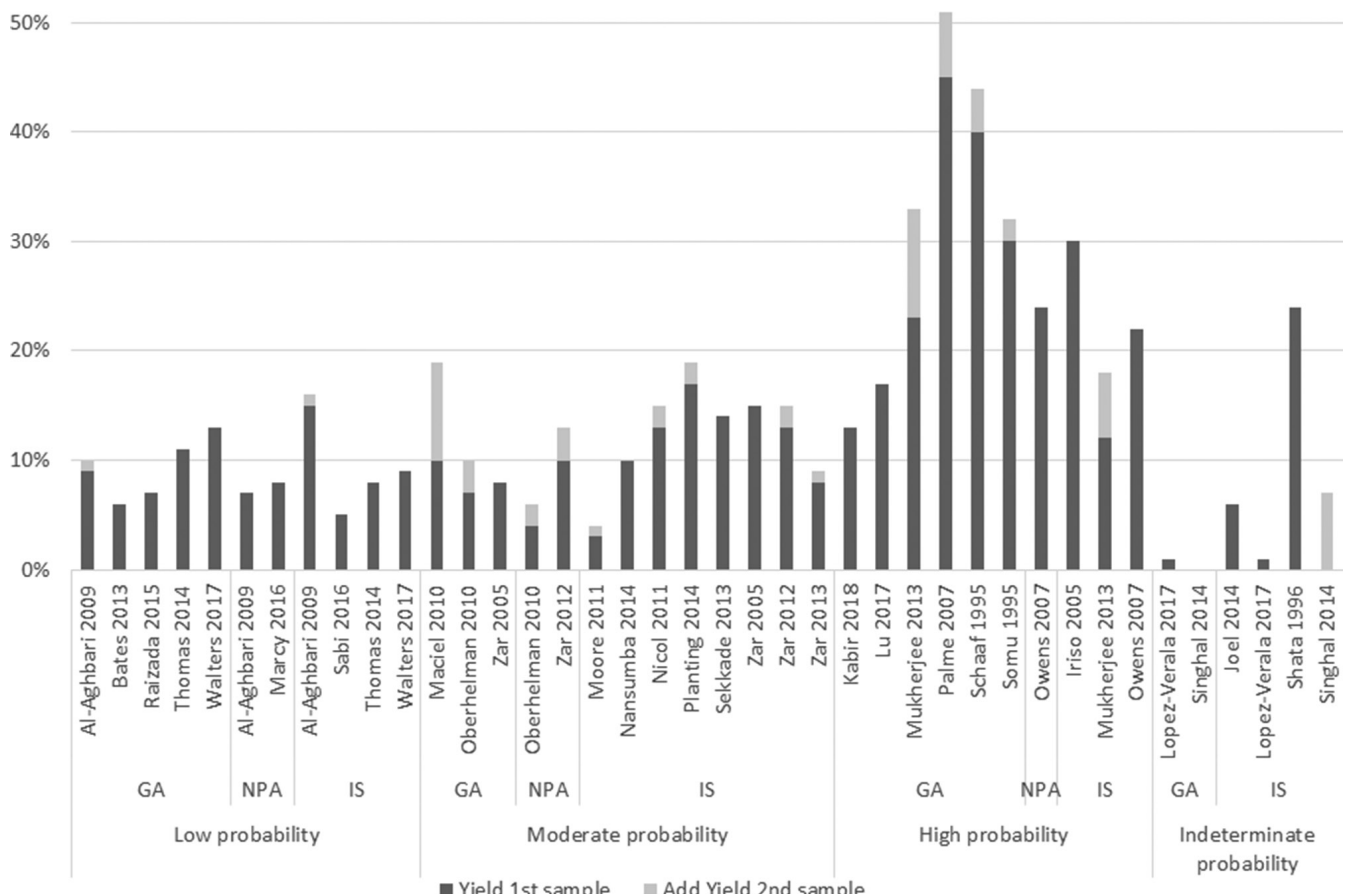

Figure 1 Yield of culture according to specimen collection method and pretest probability of intrathoracic tuberculosis. GA, gastric aspirate; IS, induced sputum; NPA, nasopharyngeal aspirate.

definition of presumptive TB. In children with low pretest probability, yields are low and IS tends to detect more cases than GA and NPA. In children with high pretest probability, detection yields tend to be better with GA. These trends are more obvious in sufficiently powered studies comparing head to head IS and GA, like in the Zar $e a^{29}{ }^{29}$ and Mukherjee $e t a l^{33}$ studies. Although

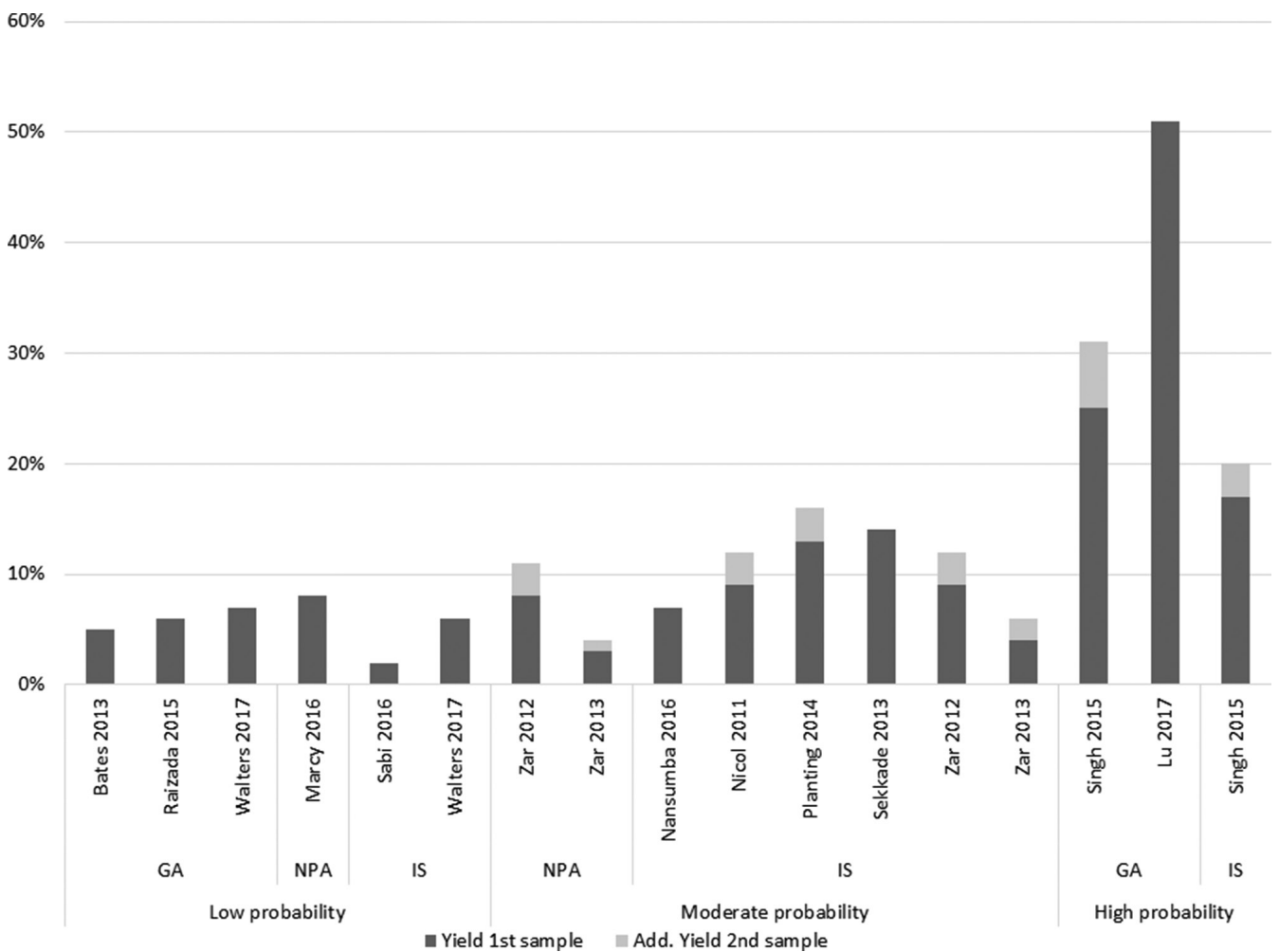

Figure 2 Yield of Xpert MTB/RIF according to specimen collection method and pretest probability of intrathoracic tuberculosis. GA, gastric aspirate; IS, induced sputum; NPA, nasopharyngeal aspirate. 
Table 3 Yield of culture and Xpert MTB-RIF with combination of different specimen collection methods and contribution of each method

\begin{tabular}{|c|c|c|c|c|c|c|c|}
\hline \multirow[b]{2}{*}{ Study } & \multirow[b]{2}{*}{ Pretest probability } & \multirow{2}{*}{$\begin{array}{l}\text { Specimen collection } \\
\text { strategy (test) }\end{array}$} & \multirow{2}{*}{$\begin{array}{l}\text { Yield of combinations, } \\
\mathrm{n} / \mathrm{N}(\%)\end{array}$} & \multirow{2}{*}{$\begin{array}{l}\text { Yield of combinations } \\
\text { on first day, } n(\%)\end{array}$} & \multicolumn{2}{|c|}{ Contribution per method* } & \multirow[b]{2}{*}{ IS, n (\%) } \\
\hline & & & & & GA, n (\%) & NPA, n (\%) & \\
\hline Al-Aghbari et $\left.a\right|^{5}$ & Low & $\begin{array}{l}\text { 1NPA+3GA+3 IS/ES } \\
\text { (culture) }\end{array}$ & 29/213 (13.6) & $29(100)$ & $19(66)$ & $14(48)$ & 27 (93) \\
\hline Mukherjee et a ${ }^{\beta 3}$ & High & 2GA+2IS (culture) & $147 / 403(36.5)$ & $109(74)$ & $131(89)$ & & $72(49)$ \\
\hline Singh et $a l^{14}$ & High & 2GA+2IS (Xpert) & $49 / 130(38)$ & $40(81)$ & $41(84)$ & & $29(59)$ \\
\hline Oberhelman et al $l^{7}$ & Moderate & 2GA+2NPA (culture) & $22 / 210(10.5)$ & NA & $22(100)$ & $12(55)$ & \\
\hline Owens et $a l^{8}$ & High & 1NPA+1IS (culture) & $24 / 88(27.3)$ & $24(100)$ & & $21(87.5)$ & $19(79)$ \\
\hline Zar et al ${ }^{29}$ & Moderate & 3GA+3IS (culture) & $58 / 250(23.2)$ & NA & $38(66)$ & & $51(88)$ \\
\hline Zar et $a l^{37}$ & Moderate & 2NPA+2IS (culture) & 63/396 (15.9) & NA & $48(76)$ & & $60(95)$ \\
\hline Zar et al ${ }^{19}$ & Moderate & 2NPA+2IS (Xpert) & $51 / 396(12.9)$ & NA & $41(80)$ & & $45(88)$ \\
\hline
\end{tabular}

*Number of children with a positive test per specimen collection method divided by the total number of children with a positive test by combination of methods.

ES, expectorated sputum; GA, gastric aspirate; IS, induced sputum; NA, not applicable; NPA, nasopharyngeal aspirate.

the performance of the IS and GA might be affected differently by the pretest probability of ITB, other factors could explain the variation like the difference in age, HIV prevalence, level of health facility and experience of the study personnel with different methods. The review shows the benefit of collecting two specimens instead of one. This often requires repeating the collection over two consecutive days, which can be challenging for outpatients and for settings with limited bed capacity. However, we also report that collecting two different specimens on the first day could be a good alternative.

This review has several limitations. (1) The absence of reference standard to diagnose ITB in children precludes attempt to compare accuracy of the methods. (2) Only few studies had appropriate design to compare yields of sputum collection methods in paired samples. ${ }^{14} 19202933$ (3) We did not include string test in our review due to the few number of studies and its poor applicability to young children who cannot swallow the device. ${ }^{3134}$ (4) Due to study heterogeneity, particularly in the definition of 'clinical suspicion of ITB', we could not perform a meta-analysis.(5) Most studies were conducted in tertiary care centres limiting the generalisability of the results to lower centres. The generalisability of the results is also limited by the fact that two-thirds of the study participants were from South-Africa or India. (6) Very few data was available on feasibility aspects of the methods, which is crucial for their use in primary care centres of LMIC.

Both IS and GA are recommended by WHO for diagnosis of ITB in children unable to produce sputum. ${ }^{36}$ However, in most LMIC, these methods are poorly implemented, especially at secondary or primary healthcare settings due to operational challenges. ${ }^{37}$ IS requires adequate training of health workers, equipment and hygiene precautions and GA requires admission of the child. Improving diagnostic capacity at secondary and primary healthcare centres is crucial to increase case detection. NPA might be an easier and better accepted method compared with IS or GA, but its implementation would require optimisation of equipment (aspirator) and material (mucus extractor). Recently, a great interest emerged from the use of stool to detect TB bacilli that are swallowed for the diagnosis of childhood TB. Despite variable sensitivity of Xpert on stools (32\%-85\%) between studies, and laboratory challenges to find the best sample processing method to remove the PCR inhibiting factors common in stool, this specimen could be a good alternative to sputum in children. ${ }^{3138-42}$ Authors have shown that Xpert on the combination of stool and NPA could achieve similar detection yields as 2SI or 2GA, offering a good opportunity for decentralisation of the microbiological diagnosis of childhood TB. ${ }^{3143}$ The use of Xpert Ultra cartridge that has a level of detection close to the one of culture with the upcoming GeneXpert Omni Point of Care platform combined to more child friendly specimens collection methods like NPA and stools could significantly improve the microbiological diagnostic capacity of childhood ITB in LMIC but the logistic implications in programmatic conditions will need to be assessed. ${ }^{44}$

Contributors VI designed the study, wrote the study protocol, performed the study screening, selection and data collection as first reviewer; performed the statistical analysis and wrote the manuscript. $\mathrm{HC}$ performed the study screening, selection and data collection as second reviewer and revised the manuscript. MB supervised the research work and was the third reviewer in case of discordance between $\mathrm{VI}$ and $\mathrm{HC}$ during study screening and selection and revised the manuscript.

Funding The study was part the work of a Master of Epidemiology (London School of Hygiene and Tropical Medicine) and was funded by Médecins Sans Frontières.

Competing interests None declared.

Patient consent Not required.

Provenance and peer review Not commissioned; externally peer reviewed.

\section{REFERENCES}

1 Perez-Velez CM, Marais BJ. Tuberculosis in children. N Engl J Med 2012;367:348-61.

2 Detjen AK, DiNardo AR, Leyden J, et al. Xpert MTB/RIF assay for the diagnosis of pulmonary tuberculosis in children: a systematic review and meta-analysis. Lancet Respir Med 2015:3:451-61.

3 Newton SM, Brent AJ, Anderson S, et alLancet Infect Dis 2008;8:498-510.

4 WHO. WHO | Global tuberculosis report 2017: WHO, 2017. http://www.who.int/tb/ publications/global_report/en/. (accessed 18 Apr 2018).

5 Al-Aghbari N, Al-Sonboli N, Yassin MA, et al. Multiple sampling in one day to optimize smear microscopy in children with tuberculosis in Yemen. PLoS One 2009;4:e5140.

6 Bates M, O'Grady J, Maeurer M, et al. Assessment of the Xpert MTB/RIF assay for diagnosis of tuberculosis with gastric lavage aspirates in children in sub-Saharan Africa: a prospective descriptive study. Lancet Infect Dis 2013;13:36-42.

7 Oberhelman RA, Soto-Castellares G, Gilman RH, et al. Diagnostic approaches for paediatric tuberculosis by use of different specimen types, culture methods, and PCR: a prospective case-control study. Lancet Infect Dis 2010;10:612-20.

8 Owens S, Abdel-Rahman IE, Balyejusa S, et al. Nasopharyngeal aspiration for diagnosis of pulmonary tuberculosis. Arch Dis Child 2007;92:693-6.

9 Berggren Palme I, Gudetta B, Bruchfeld J, et al. Detection of Mycobacterium tuberculosis in gastric aspirate and sputum collected from Ethiopian HIV-positive and HIV-negative children in a mixed in- and outpatient setting. Acta Paediatr 2004;93:311-5.

10 Planting NS, Visser GL, Nicol MP, et al. Safety and efficacy of induced sputum in young children hospitalised with suspected pulmonary tuberculosis. Int J Tuberc Lung Dis 2014;18:8-12.

11 Qureshi UA, Gupta AK, Mahajan B, et al. Microbiological diagnosis of pulmonary tuberculosis in children: comparative study of induced sputum and gastric lavage. Indian J Pediatr 2011;78:1429-30.

12 Raizada N, Sachdeva KS, Swaminathan S, et al. Piloting upfront Xpert MTB/RIF tsting on various specimens under programmatic conditions for diagnosis of TB \& DR-TB in paediatric population. PLoS One 2015;10:e0140375. 
13 Sekadde MP, Wobudeya E, Joloba ML, et al. Evaluation of the Xpert MTB/RIF test for the diagnosis of childhood pulmonary tuberculosis in Uganda: a cross-sectional diagnostic study. BMC Infect Dis 2013;13:133.

14 Singh S, Singh A, Prajapati S, et al. Xpert MTB/RIF assay can be used on archived gastric aspirate and induced sputum samples for sensitive diagnosis of paediatric tuberculosis. BMC Microbiol 2015;15:191.

15 Singhal KK, Sethi GR HM. Comparison of yield of acid fast bacilli (AFB) from induced sputum versus gastric lavage for diagnosis of pulmonary tuberculosis in children. Eur Respir Journal Conf Eur Respir Soc Annu Congr 2014 Munich Ger 2014.

16 Somu N, Swaminathan S, Paramasivan CN, et al. Value of bronchoalveolar lavage and gastric lavage in the diagnosis of pulmonary tuberculosis in children. Tuber Lung Dis 1995;76:295-9.

17 Iriso R, Mudido PM, Karamagi C, et al. The diagnosis of childhood tuberculosis in an HIV-endemic setting and the use of induced sputum. Int J Tuberc Lung Dis 2005:9:716-26.

18 Thomas TA, Heysell SK, Moodley P, et al. Intensified specimen collection to improve tuberculosis diagnosis in children from Rural South Africa, an observational study. BMC Infect Dis 2014;14:11

19 Zar HJ, Workman L, Isaacs W, et al. Rapid molecular diagnosis of pulmonary tuberculosis in children using nasopharyngeal specimens. Clin Infect Dis 2012;55:1088-95.

20 Zar HJ, Workman L, Isaacs W, et al. Rapid diagnosis of pulmonary tuberculosis in African children in a primary care setting by use of Xpert MTB/RIF on respiratory specimens: a prospective study. Lancet Glob Health 2013:1:e97-e104.

21 Sabi I, Kabyemera R, Mshana SE, et al. Pulmonary TB bacteriologically confirmed by induced sputum among children at Bugando Medical Centre, Tanzania. Int J Tuberc Lung Dis 2016;20:228-34.

22 Schaaf HS, Beyers N, Gie RP, et al. Respiratory tuberculosis in childhood: the diagnostic value of clinical features and special investigations. Pediatr Infect Dis J 1995;14:189-94.

23 Shata AM, Coulter JB, Parry CM, et al. Sputum induction for the diagnosis of tuberculosis. Arch Dis Child 1996;74:535-7.

24 Kabir S, Uddin MKM, Chisti MJ, et al. Role of PCR method using IS6110 primer in detecting Mycobacterium tuberculosis among the clinically diagnosed childhood tuberculosis patients at an urban hospital in Dhaka, Bangladesh. Int J Infect Dis 2018;68:108-14.

25 López-Varela E, García-Basteiro AL, Augusto OJ, et al. High rates of non-tuberculous mycobacteria isolation in Mozambican children with presumptive tuberculosis. PLoS One 2017;12:e0169757.

26 Lu J, Li H, Dong F, et al. The feasibility of Xpert MTB/RIF testing to detect rifampicin resistance among childhood tuberculosis for prevalence surveys in Northern China. Biomed Res Int 2017;2017:1-10.

27 Walters E, van der Zalm MM, Palmer M, et al. Xpert MTB/RIF on stool Is useful for the rapid diagnosis of tuberculosis in young children with severe pulmonary disease. Pediatr Infect Dis J 2017;36:837-43.

28 Joel DR, Steenhoff AP, Mullan PC, et al. Diagnosis of paediatric tuberculosis using sputum induction in Botswana: programme description and findings. Int I Tuberc Lung Dis 2014;18:328-34.
29 Zar HJ, Hanslo D, Apolles P, et al. Induced sputum versus gastric lavage for microbiological confirmation of pulmonary tuberculosis in infants and young children: a prospective study. Lancet 2005;365:130-4.

30 Maciel EL, Peres RL, do Prado TN, et al. Saline nebulization before gastric lavage in the diagnosis of pulmonary tuberculosis in children and adolescents. J Trop Pediatr 2010;56:458-9.

31 Marcy O, Ung V, Goyet S, et al. Performance of Xpert MTB/RIF and alternative specimen collection methods for the diagnosis of tuberculosis in HIV-infected children. Clin Infect Dis 2016;62:1161-8.

32 Moore HA, Apolles P, de Villiers PJ, et al. Sputum induction for microbiological diagnosis of childhood pulmonary tuberculosis in a community setting. Int J Tuberc Lung Dis 2011;15:1185-90.

33 Mukherjee A, Singh S, Lodha R, et al. Ambulatory gastric lavages provide better yields of Mycobacterium tuberculosis than induced sputum in children with intrathoracic tuberculosis. Pediatr Infect Dis J 2013;32:1313-7.

34 Nansumba M, Kumbakumba E, Orikiriza $P$, et al. Detection yield and tolerability of string test for diagnosis of childhood intrathoracic tuberculosis. Pediatr Infect Dis $J$ 2016;35:146-51.

35 Nicol MP, Workman L, Isaacs W, et al. Accuracy of the Xpert MTB/RIF test for the diagnosis of pulmonary tuberculosis in children admitted to hospital in Cape Town, South Africa: a descriptive study. Lancet Infect Dis 2011;11:819-24.

36 WHO. Guidance for national tuberculosis program on the management of tuberculosis in children: Gen, 2014. http://tbevidence.org/wp-content/uploads/2014/04/WHOGuidance-Childhood-TB-2014.pdf. (accessed 27 Sep 2014).

37 Reid MJ, Saito S, Fayorsey R, et al. Assessing capacity for diagnosing tuberculosis in children in sub-Saharan African HIV care settings. Int J Tuberc Lung Dis 2012:16:924-7.

38 Walters E, Demers AM, van der Zalm MM, et al. Stool culture for diagnosis of pulmonary tuberculosis in children. J Clin Microbiol 2017;55:3355-65.

39 Walters E, Gie RP, Hesseling AC, et al. Rapid diagnosis of pediatric intrathoracic tuberculosis from stool samples using the Xpert MTB/RIF Assay: a pilot study. Pediatr Infect Dis J 2012;31:1316

40 Nicol MP, Spiers K, Workman L, et al. Xpert MTB/RIF testing of stool samples for the diagnosis of pulmonary tuberculosis in children. Clin Infect Dis 2013:57:e18-e21.

41 LaCourse SM, Pavlinac PB, Cranmer LM, et al. Stool Xpert MTB/RIF and urine lipoarabinomannan for the diagnosis of tuberculosis in hospitalized HIV-infected children. AIDS 2018;32:69-78

42 Banada PP, Naidoo U, Deshpande S, et al. A novel sample processing method for rapid detection of tuberculosis in the stool of pediatric patients using the Xpert MTB/RIF assay. PLoS One 2016;11:e0151980.

43 Song R, Click E, Mccarthy K, et al. Diagnostic yield of Xpert MTB/RIF assay and Mycobacterium tuberculosis culture on respiratory and non-respiratory specimens among Kenyan children. CapeTown: UnionConference, 2015. Abstract OA-440-05.

44 Dorman SE, Schumacher SG, Alland D, et al. Xpert MTB/RIF Ultra for detection of Mycobacterium tuberculosis and rifampicin resistance: a prospective multicentre diagnostic accuracy study. Lancet Infect Dis 2018;18. 